\title{
LA MEJORA DE LAS COMPETENCIAS COMUNICATIVAS EN LA FORMACIÓN INICIAL UNIVERSITARIA
}

\section{Patricia Vilcapuma Vinces}

$\mathrm{L}$

as competencias escritas y orales de los estudiantes que se inician en la vida universitaria siempre han sido una de las cuestiones que preocupan tanto a docentes como a autoridades universitarias. Es conocido que el origen de este "pequeño» inconveniente se remonta hasta la etapa escolar y se han puesto en discusiones técnicas, fórmulas y soluciones que incluso pasan el problema a los textos literarios como si estos tuvieran este único fin en su naturaleza. Es más lamentable todavía que al llegar a la etapa superior se verifique en resultados de exámenes y tareas, solicitadas por materias que exigen el manejo adecuado de un discurso, una debilidad por parte del alumno al respecto. La práctica oral y la práctica escrita pasan sin duda por la comprensión, por ello, en toda institución universitaria la atención a las competencias comunicativas debe ser prioridad desde el inicio de la carrera teniendo en cuenta que se espera perfilar al candidato a un correcto desempeño de su ejercicio profesional en todo nivel.

Es comunicadora social. Ha estudiado una maestría en Literatura y Cultura Brasileñas (UCSS-UNMSM). Ha sido coordinadora del Área de Comunicación y Producción del Lenguaje de la UCSS y corresponsable del Plan de Mejoras de las Competencias Comunicativas en la Formación Inicial docente de la Facultad de Ciencias de la Educación de la UCSS. 
Antes de explicar esta propuesta de trabajo, es importante plantear claramente qué competencia se espera en la sala de clase. No pretendo reseñar cuestiones epistemológicas ni de mercado. La práctica educativa a todo nivel trasciende estos aspectos y quien entre en ella debe mostrar al estudiante una postura fiable y debe tener la sensibilidad necesaria para provocarlo, desafiándolo constantemente a utilizar lo que ya sabe - la lucha incansable contra los conocimientos muertos- $y$, a partir de ello, encaminarlo a la reconstrucción y mejoramiento de sus capacidades.

\section{UN TRABAJO DE DOS}

Entrar en el aula de clase implica que el docente ponga a disposición de sus estudiantes, aunque no lo diga explícitamente, su cultura profesional, su historia personal y sus concepciones respecto del proceso de aprendizaje. Por su parte, el alumno trae consigo su propia experiencia — recordemos que la universidad no tiene el monopolio de la producción del conocimiento-, su propia historia de lectura —extensa o corta- y sus propios intereses. Si tenemos en cuenta que enseñar mediante competencias implica formar, desarrollar, adquirir, construir (cf. Tobón 2005: 195), el papel del docente cambia. Es decir, el saber ser, saber conocer y saber-hacer deja de ser esa fórmula tan antipática, «verdad eterna», que se repite incansable y mecánicamente sin meditar ni considerar que optar por la vía pedagógica de la competencia es mirar al alumno en función de lo que él es: los conocimientos ya adquiridos, sus representaciones, sus intereses; en suma, es tratar con una persona. Asimismo, implica organizar la práctica educativa y no dejarla a los azares de la vida; en otras palabras, es preparar a quien aprende para saber qué hacer y cuándo (y pensemos en todo lo que ello encierra), y no sólo para un «saber ejecutar», porque algunos conocimientos no se usan en otras situaciones y menos de la misma manera. Se puede decir, entonces, que se hace un camino intermedio en este proceso: «entre un aprendizaje fuera de la vida [en el aula] y un aprendizaje "en el lugar de trabajo" [en el aula]» (Denyer et ál 2007: 103) 
Por otro lado, en una pedagogía de las competencia el discurso magisterial es sustituido por una acción magisterial que consiste esencialmente en «a) orientar, rectificar hacer modelizar el proceso de resolución de la tarea [o la «orquestación de los recursos», como diría Le Boterf] y b) apostar; hacer encontrar o hacer construir, según las circunstancias, los recursos necesarios para esta resolución [recordemos que no necesariamente son nuevos los conocimientos, a menudo solamente se descubre cómo utilizar lo que ya se sabe]».

\title{
Comparación del rol del docente
}

\begin{abstract}
Rol del maestro en una
Rol del maestro en una

pedagogía transmisiva

pedagogía por competencias

Discurso magisterial

Acción del alumno

$\downarrow$

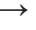

$\uparrow$

Intervenciones reguladoras-

informativas del profesor

(Denyer et ál 2007: 109)

LA PROPUESTA

Antes de empezar a trabajar es importante, como ya se señaló, ordenar la práctica, que no es otra cosa que, en primer lugar, identificar nuestras debilidades: las de los estudiantes y las de los docentes. A continuación, presentaremos las más frecuentes y más adelante explicaremos lo que atenderemos en esos puntos:
\end{abstract}

- Principales dificultades de los estudiantes

1. Problemas para distinguir el lenguaje formal del informal. 


\section{PATRICIA VILCAPUMA VINCES}

2. Desconocimiento de la importancia y significado de los paratextos. ${ }^{1}$

3. Desconocimiento de la normativa.

- Principales dificultades de los docentes

1. Separación entre la formación inicial y la formación de especialidad.

2. Falta de comunicación que lleva a diferencias de criterios (nominación y forma) en el planteamiento de tareas típicas (géneros académicos) del quehacer universitario.

3. Creencia de que la expresión académica de contenidos, tanto oral como escrita, sólo corresponde a los cursos de comunicación o producción del lenguaje.

En segundo lugar, identificar los ejes de trabajo:

- Ejes de emergencia para el inicio del trabajo

1. Luchar contra los conocimientos muertos: intervención reguladorainformativa del docente.

2. Conciencia de que todo profesor —indistintamente de la especialidad - es responsable de la adecuada producción del lenguaje de los estudiantes (en otras palabras, todas las materias son corresponsables de esta mejora)

1 Etimológicamente significa 'todo lo que está junto o alrededor del texto' (del griego para: 'junto a', 'al lado de'). Para Genette son elementos paratextuales la tapa, la contratapa, las solapas, las ilustraciones de un libro, diario o revista; los prefacios, notas al pie o al final, epígrafes, dedicatorias, índices, apéndices, bibliografía, además de los elementos tipográficos y de diseño gráfico, y hasta el formato y el tipo de papel de la edición (cf. Nogueira, coord., 2005 y Lluch 2004). En el trabajo académico también es común que los estudiantes hagan uso de elementos paratextuales para la presentación y el orden de la exposición de tareas o exámenes solicitados. 
Finalmente, una vez establecidas estas pautas las materias responsables del plan deben ser identificadas para coordinar con las corresponsables las principales necesidades de comunicación según la disciplina. Si es necesario, se revisará o reformulará los contenidos sobre los cuales trabajar. Esto llevará a proponer tareas, planes e indicadores de evaluación para que las materias corresponsables apoyen efectivamente la mejora de las competencias.

Ejemplo:

\section{Ciclos I y II (primer año)}

\begin{tabular}{|c|c|c|}
\hline Materias responsables & Contenidos & Materias corresponsables \\
\hline $\begin{array}{c}\text { Seminario de trabajo } \\
\text { intelectual }\end{array}$ & $\begin{array}{l}\text {-Mejorar la comprensión lectora. } \\
\text {-Ejercitar el pensamiento crítico. } \\
\text {-Sensibilizarse por el trabajo } \\
\text { académico y la producción } \\
\text { derivada de éste. } \\
\text {-Combatir el plagio. }\end{array}$ & $\begin{array}{l}\text { Introducción a la Filosofía } \\
\text { Seminario de realidad nacional I } \\
\text { Taller de creatividad }\end{array}$ \\
\hline $\begin{array}{l}\text { Comunicación y } \\
\text { producción del lenguaje }\end{array}$ & $\begin{array}{l}\text {-Mejorar la expresión y } \\
\text { comprensión orales } \\
\text {-Reflexionar sobre su competencia } \\
\text { lingüística. } \\
\text {-Seleccionar y utilizar } \\
\text { adecuadamente los elementos de la } \\
\text { normativa española. }\end{array}$ & $\begin{array}{l}\text { Historia universal I } \\
\text { Historia del Arte I } \\
\text { Introducción a la Biblia } \\
\text { Seminario de realidad nacional II } \\
\text { Tecnología de la informática }\end{array}$ \\
\hline $\begin{array}{c}\text { Redacción y argumentación } \\
\text { académicas }\end{array}$ & $\begin{array}{l}\text {-Organizar el discurso a nivel } \\
\text { cognitivo y prag mático. } \\
\text {-Producir textos atendiendo la } \\
\text { adecuación, cohesión y coherencia. } \\
\text {-Producir textos argumentativos. }\end{array}$ & $\begin{array}{l}\text { Introducción a la Administración } \\
\text { Taller de lectura literaria } \\
\text { Historia universal II } \\
\text { Historia del Arte II }\end{array}$ \\
\hline
\end{tabular}




\section{PATRICIA VILCAPUMA VINCES}

\section{El trabajo con los estudiantes}

Como ya se indicó líneas arriba, son tres los puntos más comunes en el trabajo académico universitario que requieren nuestra atención:

1. Problemas para distinguir el lenguaje formal del informal.

Es básico que el alumno identifique la característica de la situación comunicativa en la que participa, es decir, la académica. En ese sentido debe ajustarse al estilo formal: precisión y propiedad léxica, construcciones sintácticas correctas, exposición ordenada de sus ideas, evitar las formas coloquiales y evitar el tuteo.

Pertenecer a una institución como la universidad exige hablar y escribir adecuadamente sobre los saberes que allí se adquieren, producen, discuten o transforman. Asimismo, demanda que se comuniquen según los modos que la universidad determine. Es así que el discurso académico, propio de un ámbito universitario, tiene sus propias características. Es preciso explicar cómo abordar exitosamente la variedad de tareas asignadas al estudiante universitario a lo largo de su carrera y que se agrupan bajo la etiqueta de «trabajos académicos», que son los llamados géneros discursivos.

Los géneros discursivos son el producto de las diferentes situaciones comunicativas que se desarrollan en la actividad humana. Las personas conocen y utilizan diversos de estos géneros porque participan de diferentes prácticas sociales que implican el uso del lenguaje con funciones diversas. Por ejemplo, para comunicarse con amigos emplean géneros discursivos coloquiales, como la conversación, un saludo enviado al teléfono móvil, entre otros apropiados para relaciones interpersonales íntimas. En una situación universitaria los enunciados, productos concretos de la enunciación (de la acción de enunciar: 'expresar oralmente o por escrito algo'), responden al género discursivo académico.

Los enunciados que responden a un mismo género, en nuestro caso, el académico, tienen en común un estilo, el planteamiento claro de un 
tópico o tema y una correcta estructura o composición. Estas características permiten diferenciar el lenguaje que se empleará, por ejemplo, en una carta escrita a un amigo sobre la visita a un museo, de un informe de la visita al mismo museo dirigida a un profesor que ha solicitado esta tarea; una monografía de una novela; una exposición oral en clase de una conversación amical, entre otros. Esta identificación y comprensión de la construcción de un género discursivo que haga el estudiante y del lugar que éste debe asumir, es decir, de «enunciador académico», permiten entender que las normas a las que responde la producción académica imponen cuidados que si se obvian pueden ocasionar errores de comunicación y sanciones, como por ejemplo, presentar una tarea que, si bien corresponda a los solicitado por el docente, sea expuesta con un lenguaje coloquial o con un vocabulario que no se ajuste a la disciplina, o que el contenido sea insuficiente y tan sólo exponga los apuntes tomados en clase; o presentar la tarea sin indicar quién es o quiénes son los autores de las ideas desarrolladas en el caso de tomar textualmente alguna cita de un libro. Es evidente que la valoración que haga el docente sobre la tarea y que se expresa en un puntaje no será favorable para el alumno que no cumpla con estos requerimientos.

2. Desconocimiento de la importancia y significado de los paratextos.

En una tarea, los elementos paratextuales varían según su naturaleza. Si es una tarea de extensión mayor, es probable que recurramos a hojas de presentación, carátulas, números de página, tipografía especial. En un examen, si es manuscrito, se escogerá un color adecuado de tinta, se cuidará la distribución de lo escrito en la hoja en blanco, se subrayará, etcétera. Dado el significado del paratexto, como la relación con el contexto de su producción, no sería apropiado, en el caso de las tareas, colocar pegatinas o hacer dibujos en la carátula, o escribir en ambas caras de la hoja; en el caso de un examen, no se debe abusar de los correctores líquidos, o utilizar marcas personales para hacerle alguna indicación al docente o anotar cuestiones ajenas a las respuestas de la evaluación. 
Por otro lado, desarrollar la habilidad lectora de los elementos paratextuales favorece el desarrollo general de la competencia lectora y, sobre todo, permite al estudiante convertirse en elector de sus materiales de lectura.

A continuación ofrecemos una clasificación sobre las formas que puede asumir el paratexto en libros y tareas:

Paratexto icónico: la iconicidad define lo paratextual en general. Transmite información significativa mediante el recurso (diseño del paratexto) a la imagen. Por ejemplo, fotos, dibujos, infografías, diseño tipográfico (paciencia, paciencia, paciencia, etc.), los resaltes tipográficos (paciencia, paciencia, PACIENCIA, «paciencia», etc.), signos ortográficos para editar citas (corchetes, paréntesis, puntos suspensivos, etc.), mapas, cuadros, diagramas, formato del libro, la cubierta (carátula, contracarátula, lomo, solapas), entre otros.

Paratexto verbal: en líneas generales, son aquellos textos que acompañan al contenido para insertar el libro dentro de un contexto determinado - tanto los paratextos verbales como los icónicos convierten el texto en libro- o facilitar la lectura de la tarea o examen.

En un libro, el paratexto verbal se ubica en la tapa, contratapa, solapas y en las primeras y últimas páginas.

a. Información de la tapa: nombre del autor, título y subtítulo del libro, sello de la colección (puede no tenerlo) y sello editorial.

b. Información de la contratapa: se describen las características del texto con la finalidad de convencer al destinatario sobre las bondades del libro.

c. Información de las solapas: si el libro las tiene, a veces se encuentra una biografía breve del autor, el listado de los libros de la editorial o algún comentario sobre la obra.

d. Información de las primeras páginas: corresponde a la portadilla (generalmente va el título del libro en letra pequeña), la portada (repetición de los carátula o tapa), la hoja de créditos (titulo y 
subtítulo del libro, nombre del autor, menciones legales, cantidad de ejemplares de la tirada, etc.), índice, dedicatoria, presentación, prólogo, introducción, entre otros.

e. Información de las últimas páginas: colofón (datos relativos al trabajo de impresión), listado de las obras de la editorial, etc.

$\mathrm{Al}$ interior del libro, al igual que las tareas, se utilizan generalmente:

a. Títulos y subtítulos: son indicadores del tema y las ideas que merecen destacarse.

b. Epígrafes: citas que pueden comentar un título o un texto.

c. Citas y notas: se ubican a lo largo del texto. Las notas pueden aparecer al pie de la página o al final de cada capítulo del libro o monografía. Varían según el sistema de anotación bibliográfico que se adopte.

d. Bibliografía: puede encontrarse al final del trabajo o texto, o al final de cada capítulo.

e. Glosario: diccionario de términos técnicos que suele encontrarse al final del libro o cada capítulo.

3. Desconocimiento de la normativa.

No nos referimos solamente a las tildes y comas, sino a la relación de noción estructural de oración y puntuación; la corrección ortográfica y gramatical; el uso apropiado de las mayúsculas, etcétera.

\section{El trabajo con los docentes}

Definir los criterios para la presentación (forma y contenido) y la nominación de la tarea o género académico que se trabaje en aula es la base para que el estudiante tenga en claro qué es lo que se espera. A continuación, propondremos tres tipos de tareas o ejercicios que pueden ayudar a fortalecer y mejorar las competencias comunicativas desde las materias corresponsables: 


\section{PATRICIA VILCAPUMA VINCES}

1. Preparación de esquemas de contenido

- Como guión de exposición oral

- De comprensión lectora

Se recomienda el esquema numerado que permite visualizar la jerarquía de ideas. Ambos tipos de esquema son similares al conocido «plan de redacción», la diferencia es la extensión de los enunciados.

El primer tipo de esquema contiene la información esencial y útil para una exposición oral.

Recomendaciones:

a. Debe tener en cuenta el tiempo disponible para el desarrollo del tema.

b. Debe tener una clara estructura: ideas organizadas e ideas fundamentales.

c. Debe permitir que se puedan introducir ideas nuevas y comentarios.

d. Cuidar la escritura si es que se anotará el guion en la pizarra o se acompañará con material multimedia.

e. Es importante y serio mencionar las fuentes consultadas para preparar el tema de la exposición.

f. No olvidar que si durante la exposición se va a citar, directa o indirectamente, se debe hacer referencia al autor: «Según Cervantes,...»; «Coincidiendo con Barthes,...»; «Retomando las palabras de Benjamin,...»; entre otras frases.

El segundo tipo de ejercicio es una manera esquematizada de presentar el resultado del tomar notas de una lectura, que puede utilizarse como una tarea previa al informe de lectura.

Recomendaciones:

a. Lo primero que se debe considerar es la extensión y lo solicitado por el profesor. 
b. El docente puede indicar que, mediante un esquema de comprensión lectora, se explique una idea o un concepto desarrollado por el autor en una parte del texto estudiado.

c. A veces se pide un esquema de comprensión lectora de todo el libro o de un apartado del libro. En estos casos se debe seleccionar y sintetizar las ideas de tal manera que el esquema resulte claro y comprensible.

d. Si el esquema se utilizará como técnica para estudiar, no se debe olvidar que todo texto desarrolla un tema y alrededor de este se ordenan las ideas bajo un criterio de jerarquías. Reconocerlas facilitará la comprensión del tema.

e. A diferencia de un esquema de redacción, en el que sólo se enuncia las ideas mediante frases nominales, el esquema de comprensión lectora como tarea y como técnica de estudio permite desarrollar brevemente (sin llegar a ser un resumen) los contenidos.

\section{El resumen}

Se debe revisar que el texto presentado establezca claramente la relación lógica de las ideas tomadas en cuenta para su realización. Para ello, el estudiante deberá concentrarse en los conceptos e ideas subrayados, parafrasearlas y relacionarlas adecuadamente con el discurso identificado.

3. El informe universitario ${ }^{2}$

- El informe de una visita. ${ }^{3}$

2 Se ha consultado para la explicación teórica de este apartado Nogueira, coord. 2005: 123-134 y Botta 2004: 20-23.

3 Este tipo de informes fue planteado por el Área de Historia de la UCSS en una reunión de trabajo (Círculo interdisciplinario) de todas las áreas. Los profesores de este curso solicitaban con frecuencia «una tarea» sobre el museo o sitio arqueológico de visita por parte de los alumnos. Es importante, como ya se señaló, que los docentes se pongan de acuerdo en la nominación e identificación de las características de una tarea. 


\section{PATRICIA VILCAPUMA VINCES}

- El informe sobre una película.

- El informe de lectura: de un fragmento, de un libro, de un corpus de libros.

En este tipo de tarea, se evaluará la capacidad del estudiante en organizar la información y presentarla adecuadamente de tal manera que sustente la exposición y sugerencias hechas sobre lo que se informará.

Los informes varían en cuanto a su extensión y estructura, ya que deben adaptarse a la disciplina para la cual son escritos y, sobre todo, responder a lo solicitado por el profesor del curso.

Pueden ser de carácter argumentativo o de carácter expositivo. En el primero, se debe interpretar y analizar los hechos, además de presentar conclusiones que respondan al análisis presentado. En el segundo, sólo debe limitarse a informar sobre un tema o situación describiendo y narrando unos hechos o mostrando una serie de datos en línea con lo que queremos mostrar.

En cuanto a los dos primeros tipos de informe, se debe considerar a) Organizar las ideas en un esquema de redacción y b) Redactar el texto siguiendo el esquema de ideas y considerando las funciones globales de todo texto: introducción o inicio, cuerpo o desarrollo y síntesis o conclusión.

El informe de lectura requiere mayor atención, ya que la selección de datos y del material está guiada por un carácter más explicativo: dar cuenta de una lectura en un contexto universitario. El estudiante debe exponer la fuente (el libro o corpus de libros), pero también cuestionarla, reconstruirla históricamente y opinar (académicamente) sobre ella. Esto no quiere decir que sea un resumen o una suma de citas.

El discurso de los textos fuente puede ser introducido de manera literal, es decir, mediante citas textuales (discurso directo) o mediante paráfrasis (discurso indirecto o reformulación conceptual). Es pertinente anotar que estas dos posibilidades no son excluyentes sino complementarias.

El referente de un informe de lectura - el objeto por describir, es decir, la fuente - se puede construir a partir de diversas unidades textuales como una cita textual o apartado, un libro o un corpus (de textos). 
-Cómo preparar un informe de lectura

Hay que prestar atención a lo solicitado en el curso. El docente, muchas veces, indica el tema desde el cual debe partir la reflexión y la selección de fuentes. A continuación se anotan algunas recomendaciones.

a. En el caso de un libro, leer la referencia bibliográfica y establecer cuándo fue producido; en el caso de una cita textual o apartado, a qué libro pertenece; en el caso de un corpus de libros, identificar el eje temático para una adecuada selección de las fuentes y posterior justificación en la tarea.

b. Informarse sobre el autor (se debe buscar libros de él en la biblioteca y leer las solapas, contratapas, prólogos; navegar en Internet, etcétera). No se trata de realizar una biografía del escritor, sino de ubicar el campo de la disciplina para una mejor aproximación del texto.

c. Determinar el género discursivo a que responde el texto y cuál es su tópico. Analizar qué tipo de secuencias hay en el texto (expositiva o argumentativa) y cuál predomina. Según el caso, determinar qué pregunta se responde, qué hipótesis se argumenta, etcétera. Detectar, en correspondencia, qué respuesta se presenta o qué argumentos se sostienen.

d. La estructura del informe de un corpus puede variar según la extensión, y puede, por lo tanto, tener carátula e índice. En general, no se debe olvidar las funciones globales de un texto académico (introducción, cuerpo o desarrollo y cierre o síntesis) y de anotar correctamente la bibliografía consultada.

Retomando la organización del trabajo, se puede establecer, incluso, una tarea de evaluación final por ciclo, de tal manera que se pueda evaluar la competencia y no solamente, por ejemplo, procedimientos o conocimientos (estos pueden ser evaluados en pruebas de rutina). 


\section{PATRICIA VILCAPUMA VINCES}

\section{Los criterios e indicadores de evaluación}

Algunos indicadores que pueden ser supervisados en las materiales corresponsables y que ayudan a un seguimiento del avance de los alumnos, son los siguientes:

Competencia escrita

1. Adecuación a la situación comunicativa/ construcción enunciativa.

2. Organización de secuencias.

3. Comprensión del corpus (en el caso de consulta o utilización de fuentes para sustentar las respuestas).

4. Textualidad.

5. Ortografía y puntuación.

A partir de ellas cada materia puede plantear criterios (para corregir tareas) que ayuden al seguimiento del grupo, por ejemplo, ${ }^{4}$

4 Propuesta planteada en el Circulo interdisciplinario de la Facultad de Ciencias de la Educación para el trabajo conjunto en la mejora de las competencias comunicativas coordinado por Betty Alfaro Palacios, responsable académica. 


\begin{tabular}{|c|c|c|}
\hline CRITERIOS & INDICADORES & FRECUENCIA: SI/NO \\
\hline \multirow{4}{*}{ TEXTUALIDAD } & $\begin{array}{l}\text { Planteamiento adecuado } \\
\text { del tema. }\end{array}$ & \\
\hline & $\begin{array}{l}\text { Organización y relación } \\
\text { lógica entre ideas en todo } \\
\text { el texto. }\end{array}$ & \\
\hline & $\begin{array}{l}\text { Concordancia gramatical: } \\
\text { a) de género } \\
\text { b) de número }\end{array}$ & \\
\hline & $\begin{array}{l}\text { Uso correcto de } \\
\text { conectores }\end{array}$ & \\
\hline \multirow[b]{2}{*}{$\begin{array}{l}\text { ORTOGRAFÍA Y } \\
\text { PUNTUACIÓN }\end{array}$} & $\begin{array}{l}\text { Tildación (más de } 3 \\
\text { errores) }\end{array}$ & \\
\hline & $\begin{array}{l}\text { Uso correcto de los signos } \\
\text { de puntuación (tomar } \\
\text { en cuenta la noción } \\
\text { gramatical de oración). }\end{array}$ & \\
\hline
\end{tabular}

Competencia oral ${ }^{5}$

\begin{tabular}{|l|l|}
\hline \multicolumn{1}{|c|}{ CRITERIOS } & \multicolumn{1}{c|}{ INDICADORES } \\
\hline Expresión coherente de ideas & $\begin{array}{l}\text { Formulación de respuestas en forma ordenada, } \\
\text { lógica y con sentido. }\end{array}$ \\
\hline $\begin{array}{l}\text { Disposición para responder } \\
\text { preguntas orales de tipo } \\
\text { argumentativo }\end{array}$ & $\begin{array}{l}\text { Facilidad para intervenir en preguntas que } \\
\text { requieran opinión y criterios personales. }\end{array}$ \\
\hline
\end{tabular}

5 Trabajo propuesto por el profesor Manuel Vejarano Ingar, coordinador del área de Comunicación y Producción del Lenguaje de la UCSS, en el Circulo interdisciplinario de la Facultad de Ciencias de la Educación para el trabajo continuo en la mejora de las competencias comunicativas. 


\begin{tabular}{|l|l|}
\hline Uso de lenguaje formal & $\begin{array}{l}\text { Expresión sin rasgos propios de otra situación } \\
\text { comunicativa. }\end{array}$ \\
\hline $\begin{array}{l}\text { Aceptación de correcciones y } \\
\text { sugerencias durante la exposición }\end{array}$ & $\begin{array}{l}\text { Recibir con madurez las correcciones y desarrollar } \\
\text { la autocrítica en base a ellas }\end{array}$ \\
\hline Uso de referencias bibliográficas & Uso de citas o frases de fuentes autorizadas. \\
\hline
\end{tabular}

Del seguimiento que se haga, dependerá que las materias responsables ajusten o realicen actividades de intervención en beneficio de los estudiantes, lo cual repercutirá en su desempeño académico. Para ello, es indispensable que se comparta la información obtenida con la evaluación de los indicadores propuestos, así como del progreso en los quehaceres académicos de los alumnos.

\section{LA CONTINUIDAD}

Una característica de enseñar en términos de competencia es el diálogo permanente entre el alumno y su formación continua, es decir, hacer surgir en él una reflexión sobre lo que necesita aprender o mejorar de la administración de los recursos que posee. Por otro lado, recordemos que no necesariamente lo que es motivador para nosotros lo es para el joven; así que dependerá mucho más de nuestra creatividad para plantear la tarea, que del método, conseguir su sensibilidad hacia los conocimientos gratia sui. Conformarnos a un tipo de relación con el saber (formulas de transmisión) encierra al alumno y lo condena al conocimiento muerto.

Por otro lado, pensar que las competencias comunicativas solamente son «competencia» de las materias relacionadas al lenguaje o sólo se terminan en los primeros ciclos (estudios generales) como si fuera una cuestión de moda el elaborar un currículo con cursos de lengua, redacción o de lectura es bloquear todo un camino profesional. $O$ peor aún pensar que estos cursos, como las humanidades, sólo son propios de las facultades de letras 
es demostrar una limitación y miedo por la capacidad crítica. El trabajo por competencias, o mejor dicho, el recuperar el ars curiosandi, educar la sensibilidad del joven a los conocimientos gratuitos o al placer de aprender, es la principal tarea de cualquier docente. 


\section{BIBLIOGRAFÍA}

Botтa, Mirtha

2004 Tesis. Monografías e informes. Buenos Aires: Biblos.

DenYer, Monique et ál

2007 Las competencias en la educación. Un balance. México, D.F.: Fondo de Cultura Económica.

LLUCH, Gemma

2004 ¿Cómo analizar textos infantiles y juveniles? Bogotá: Norma.

Nogueira, Sylvia (coord.)

2005 Manual de lectura y escritura universitarias: prácticas de taller. 3. ${ }^{\mathrm{a}} \mathrm{ed}$. Buenos Aires: Biblos.

TOBÓn, Sergio

2004 Formación basada en competencias. Bogotá: Ecoe Ediciones. 